\title{
Determinants of Therapy Failure Among Adults on First-line Antiretroviral Therapy in Asmara, Eritrea: A Multicenter Retrospective Matched Case-control Study
}

Samuel Tekle Mengistu ( $\nabla$ teklesam7@gmail.com )

Nakfa Hospital, Ministry of health Northern Red Sea branch, Nakfa, Eritrea

Ghirmay Ghebrekidan Ghebremeskel

Nakfa Hospital, Ministry of health Northern Red Sea branch, Nakfa, Eritrea

Hermon Berhe Ghebrat

Af'abet Hospital, Ministry of health Northern Red Sea branch, Afabet, Eritrea

Oliver Okoth Achila

Orotta college of medicine and health sciences

Nahom Asmerom Yohannes

Gedem Naval hospital

Amon Solomon Ghebrenegus

Hazhaz Zonal Referral Hospital

Filimon Gebretsadik

Halibet National Referral Hospital

Naod Yeibyo

Ghindae Zonal Referral Hospital, Ministry of health Northern Red Sea branch, Ghindae, Eritrea

Amanuel Kidane Andegiorgish

Xi'an Jiaotong University Health Science Center

\section{Araya Berhane Mesfn}

Communicable disease control division, Ministry of health, Eritrea

Negassi Leake

Orotta College of Medicine and health sciences

\section{Research Article}

Keywords: CD4+ cell count, HIV, Treatment failure, Viral load

Posted Date: January 11th, 2022

DOI: https://doi.org/10.21203/rs.3.rs-1200246/v1 
License: (c) (i) This work is licensed under a Creative Commons Attribution 4.0 International License. Read Full License 


\section{Abstract}

Background Information on treatment failure (TF) in People living with HIV in data-poor jurisdictions is necessary to counter the rapidly escalating epidemic of TF to first-line combined anti-retroviral therapies (cART) in sub-Saharan Africa (SSA). In this study, we examined the risk factors associated with TF in Asmara, Eritrea.

Methods: A multicenter, retrospective 1:2 matched (by age and gender) case-control study was conducted in four major hospitals in Asmara, Eritrea on adults aged $>15$ years who were on treatment for at least 6 months. Cases were patients with viral load $\geq 1000$ copies $/ \mathrm{mL}$ anytime between 2019-2021 and/or patients switched to second line cART. Controls were randomly selected from patients on first-line ART with viral load $<1000$ copies $/ \mathrm{mL}$. Data was extracted using a checklist from the master data set and analyzed using SPSS version 26. Multivariable logistic regression analysis was conducted to identify risk factors for TF. All $p$-values were 2-sided and the level of significance was set at $p<0.05$ for all analyses.

Results: Of the 1068 participants, 585 (54.7\%) were females. The median age at treatment initiation was 46 years (interquartile range (IQR): $39-51$ ). Median time to combined antiretroviral therapy (cART) failure was 37 months (IQR =24-47). In multivariate analysis factors associated with increased likelihood of virologic failure (VF) were the type of initially used nucleoside reverse transcriptase inhibitors (NRTI) backbone ( (Zidovudine+Lamivudine (AZT+3TC): adjusted odds ratio (aOR): 2.70; 95\% Confidence interval (Cl): 1.65-4.41, p-value<0.001), (Abacavir+lamivudine (ABC+3TC): aOR: 4.73; 95\%Cl: 1.18-18.92, pvalue $=0.028$ ), and (Stavudine+Lamivudine (D4T+3TC): aOR: 5.00; 95\% Cl: 3.03-8.20, p-value<0.001), prior exposure to ART (aOR: 2.28; 95\% Cl:1.35-3.86; $\mathrm{p}=0.002$ ), record of sub-optimal drug adherence (aOR: 3.08; 95\%Cl: 2.22-4.28; $\mathrm{p}<0.001$ ), ambulatory/bedridden at presentation (aOR:1.61; 95\%Cl: 1.12-4.28; $\mathrm{p}-$ value $=0.010$ ), presence of comorbidities (aOR: $2.37 ; 95 \% \mathrm{Cl}: 1.36-4.10$, $\mathrm{p}$-value $=0.002$ ), duration of cART (<5 years: aOR: $5.90 ; 95 \% \mathrm{Cl}: 3.95-8.73$, p-value $<0.001$ ), and use of SMX-TMP prophylaxis (aOR : 2.00, $95 \% \mathrm{Cl}, 1.44-2.78$, p-value $<0.001)$.

Conclusion: Our findings underscore the importance of optimizing CART adherence, diversification of CART regimens, and interventions directed at enhancing early HIV diagnosis, prompt initiations of treatment and improved patient focused monitoring of treatment response.

\section{Key Summary Points}

\section{Why carry out this study?}

- Very brief background leading to the study, including for example disease population,

- economic burden and/or unmet need. (1-2 bullet points)

Lack of data regarding magnitude and possible risk factors for TF in Eritrea make it difficult to make goal-directed improvements in patient-specific care. This study has identified important factors that impact therapy outcome negatively, it is our considered opinion that identifying such patients and 
implementing a targeted viral load/CD4 ${ }^{+}$cell count monitoring will be paramount to tackling this merging challenge.

- What did the study ask? /What was the hypothesis of the study? (1 bullet point)

\section{o The study inquired the magnitude of TF, sociodemographic and clinical risk factors for TF What was learned from this study?}

- What were the study outcomes/conclusions? (data) (1 bullet point)

The study revealed high burden of TF and important clinical risk factors for poor therapy outcome

- What has been learned from the study? This can be any outcome even if it contradicts the

- initial study hypothesis. If the findings were negative, neutral or purely confirmatory, how

- might this affect research and/or treatment in future? (1-2 bullet points)

The study indicates that treatment response is a multifactorial outcome and control of this epidemic calls for a multifaceted approach to tackle. i.e improvements need to be emphasized in multiple sectors of health care system with the aim of early diagnosis, prompt treatment initiation and targeted monitoring in addition to further research into the determinants of sub-optimal adherence.

\section{Introduction}

In 2020, approximately 37.7 million (95\% Cl: 30.2 million-45.1 million) people globally were living with the Human Immunodeficiency Virus (HIV) [1] of which Adults constituted 36.2 million (95\% Cl: 30.2 million 42.5 million). The data also demonstrates that a disproportionate number of 25.7 million ( 69.5\%) people living with HIV (PLWHIV) are in (SSA). In terms of mortality, approximately 36.3 million [27.2 million - 47.8 million] people have died of HIV/AIDS-related illnesses or complications in the last 3 decades [1], and a disproportionate number occurred in SSA. Fortunately, cARTs coupled with advances in HIV testing technology has transformed the HIV landscape from a disease with significant long-term morbidity and mortality (2000 - 2015, 8 million HIV-related deaths were averted) into a relatively manageable chronic condition. For context, massive global expansion of cART coverage (from 7\% (7.8 million [6.9 million-7.9 million]) in 2010 to $77 \%$ ( 27.5 million [26.5 million-27.7 million]) in 2020) [1] has led to tremendous gains in adult life expectancy. By reducing community viral load (CVL), large-scale CART rollout can also reduce HIV transmission at the population level [2]. Based on these findings, a 2015 update of the World Health Organization (WHO) treatment guideline recommended treatment for all PLHIV regardless of CD $4^{+}$cell status [3].

Notwithstanding the observed gains, the downward trend in HIV-associated mortalities appears to have stalled in recent years. Globally, HIV has still been associated with approximately 680000 [480 000-1.0 
million] mortalities per year and remains a leading cause of death in specific demographic groups or countries, particularly in SSA [4]. Multiple explanations have been invoked to explain this intractably high mortality rate [2]. However, treatment failure (defined either as clinical failure - AIDS-defining event; virologic failure - Failure to achieve or maintain two consecutive plasma viral loads (VL) $<1000 \mathrm{c} / \mathrm{mL}$ (a WHO standard commonly used in Low and medium-income countries (LMIC) in SSA); or immunologic failure $-\mathrm{CD} 4^{+}$cell count $<200 \mathrm{~mm}^{3}$ ) is regarded by most experts as the most formidable problem. Others have warned that the rapidly evolving epidemic of CART failure may profoundly undermine existing public health gains [5]. For context, modeling studies predicted that drug resistance mutations (DRMs) to non-nucleoside reverse transcriptase inhibitors (NNRTI) $\otimes 10 \%$, could account for 105000 and 135000 excess HIV infection and deaths in SSA between 2016 to 2020 [6]. Further, estimates suggest that by 2030, 2 million HIV patients in SSA will be on the costlier second-line cART [1].

Before 2017, most studies in SSA relied on clinical and immunological failure to evaluate treatment failure [7]. This was largely due to a lack of access to viral load testing. However, studies have shown that the sensitivity and specificity of the WHO-based immunological and clinical failure algorithm for the determination of treatment failure are low [8]. Moreover, evidence suggests that immunological and clinical failure often lags behind virologic failure [9]. For these reasons, virologic failure is regarded as the gold standard for the assessment of treatment failure [10]. Apart from the diagnostic utility, research shows that virologic failure compounds the problem of drug resistance mutations (DRMs) and limits treatment options. The major determinants of virologic failure include a combination of factors - patient factors (sub-optimal adherence to cART - psychosocial factors, co-morbidities, poor linkage to care, interruption of cART, cost, adverse drug reactions/tolerability); viral factors (persistence of drug-resistant strains (DRS), innate resistance to specific classes of drugs (e.g. CC-chemokine receptor (CCR5) antagonists in patients with syncytium-inducing SI/T-tropic variants which utilize CXC chemokine receptor CXCR4 or dual tropic strains); secondary resistance/prior failures, higher pre-treatment viral load); and drug-related factors (sub-optimal pharmacokinetics/pharmacodynamics; low genetic barrier (e.g. ARVs which require a limited number of mutations for developments of resistance - lamivudine (3TC), emtricitabine (FTC), and Efavirenz (EFV), among others), inappropriate cART regimen; Drug-drug interactions (DDI) with non-ARVs, among others) $[9,11]$. In general, varying permutations and combinations of these factors have been uncovered by investigators in SSA $[8,11]$.

Although access to data on virologic failure from SSA has improved in recent years; information from a large number of countries is missing. More importantly, program level differences, such as site-level processes or cultural differences and variations in policies and implementation priorities; limits extrapolation of data to other jurisdictions. For context, Eritrea, for instance, is regarded as one of the success stories in SSA when it comes to HIV/AIDS prevention - current estimates suggest that the prevalence of HIV is approximately $0.7 \%$ [12]. However, concerns remain regarding patients' management. Unlike other countries in the region, basic data on treatment failure or other HIV-related themes is unavailable in publicly available repositories. Therefore, the objective of this study was to examine the risk factors associated with virologic failure in Asmara, Eritrea. It's our opinion that understanding 
correlates of first-line treatment failure is essential for patient monitoring and for targeted intervention $[13,14]$. We also presented data on many important measures related to patient baseline and follow-up characteristics of PLWHA.

\section{Methodology}

\section{Study design and Setting}

A retrospective case-control study was carried out to identify the factors association with virologic failure among adults on first-line antiretroviral therapy.

This study was conducted in four major hospitals (Orotta National Referral Hospital (ONRH); Halibet National Referral hospital (HRH); Sembel hospital (SH); and Haz Haz Zonal Referral Hospital $(\mathrm{HzH})$ located in Asmara, the capital city of Eritrea. Medical records of all 6548 (inpatient and outpatients) patients who visited the aforementioned hospitals from 2001 - 2020 were collected after obtaining formal letter from the CDC office and the respective hospital. A total of 6548 patients, i.e. 2499 in ONRH, 1638 in $\mathrm{HRH}, 1361$ in $\mathrm{HzH}$, and 1050 in SH have accessed treatment for 19 years (2001-2020), see Figure 1. The four hospitals are the main treatment centers for HIV before the decentralization of service later into other five zones of the country.

\section{Study population}

Adult patients ( $\geq 18$ years of age) who received first-line cART treatment for a period of more than 6 months at one of the four major hospitals and who failed the first-line cART from 2001 - 2020 were eligible for recruitment. Participants with incomplete record were excluded from the analysis. Cases were patients with virologic failure. All incidence from 2019-2021 or drug switching due to therapy failure in the first-line regimens were included. For censoring, the first incidence of a drug change from the first-line CART to second line regimen was targeted. Controls were patients on first-line treatment and plasma viral load $<1000 \mathrm{copies} / \mathrm{ml}$ in their entire follow-up duration. For each case two matched controls were enrolled based on the plasma viral load.

\section{Sample size determination and sampling procedure}

We extracted all records from the registry from year 2001 to 2020. A total of 356 patients from the four hospitals $(\mathrm{ONRH}=124, \mathrm{HH}=95, \mathrm{HzH}=80$, and $\mathrm{SH}=57)$ with confirmed treatment failure to first-line regimens met the inclusion criteria. Each case was then matched by lottery method with two controls of similar gender and age at treatment initiation in a \pm 5 -year range. Ultimately $248,194,144$, and 130 HIVpositive patients on first-line treatment with no history of treatment failure were included as controls from each hospital respectively (Figure 1).

\section{Data collection tool and procedure}


Data was collected via a checklist from the registry database that is routinely filled in a special form for every patient upon enrollment and follow-up. The checklist was structured in a systematic way that would enable data collectors to retrieve data in an orderly fashion and detect systematic errors. All information properly labeled by the HIV-Unit in the four hospitals as per the ICD-10 standard [International classification for treatment failure] were extracted using the checklist. A team of 17 professional including; three principal investigators, four supervisors, and ten registered nurses have conducted data extraction. Training was provided for the study team before the start of data collection. Onsite routine data monitoring for completeness was conducted with a regular checklist by the principal investigators throughout the data collection period from January 2021 to May 2021. Double data entry was employed using CSPro 7 for quality control.

\section{Operational definition and study outcome}

The primary outcome of this study was to determine the factors associated with virologic failure. Moreover, we attempted to describe patient's demographic characteristics (sex, age, address, educational status, marital status, employment, and care entry point), and baseline characteristics (prior exposure to ART, baseline $\mathrm{CD} 4^{+}$cell count, WHO clinical stage, Initial CART backbone, and baseline functional status) as secondary outcome. Additional exploratory variables including TB status, CART changes, Cotrimoxazole prophylaxis, comorbid conditions, missed hospital appointments, duration on CART, weight shift (enrollment weight from current weight), low-level viremia, persistent viremia, previous hospitalizations, and their adherence were also explored. Sub-optimal adherence record comprised of any participant who had at least one record of poor or fair adherence. Late presentations is defined as $\leq 350$ $\mathrm{CD} 4^{+}$cells/ $\mu \mathrm{L}$ and/or AIDS-defining disease in WHO stage III/IV [15].

\section{Data processing and analysis}

All analyses were conducted using SPSS version 26 for windows (SPSS Inc., Chicago, Illinois, USA). Where appropriate, demographic and HIV-related characteristics of patients were summarized using percentages, medians $( \pm(\mathrm{IQR}))$, or mean \pm standard deviation (SD). Descriptive analyses were stratified by therapy outcome in all key variables at baseline using Pearson's Chi-square test or Fisher's exact test, and Mann-Whitney $\mathrm{U}$ test for continuous data. Normality tests were performed before running any statistical computations. $\mathrm{CD} 4^{+}$cell count recovery rate was calculated using the median rate of increase from treatment initiation to 6 months, 6 -12 months; 12 - 24 months, and 24 - 36 months of treatment. Nonparametric test (Mann-Whitney $U$ test) was used to compare $\mathrm{CD} 4^{+}$cell counts as well as $\mathrm{CD} 4^{+}$ recovery rates between cases and controls in the specified periods. Multivariate logistic regression (Backward: Conditional) analysis was subsequently undertaken - only variables with a $p$-value of $\leq 0.25$ in the bivariate analysis were included in the model. The potential for collinearity was minimized by not including pairs of variables with a Spearman $r>0.60$ in the same model. Results are presented as crude odds ratios (COR) and aOR with $95 \% \mathrm{Cls}$. A p-value $<0.05$ was considered as significant.

\section{Results}


Table 1 Baseline and clinical characteristics of study participants 


\begin{tabular}{|c|c|c|c|c|c|}
\hline Characteristics & $\mathbf{N}(\%)$ & Cases & Controls & Chi-square (ロ2) & $P$ value \\
\hline \multicolumn{6}{|l|}{ Gender } \\
\hline Male & $483(45.2)$ & $161(44.1)$ & $322(45.2)$ & \multirow[t]{2}{*}{0.0} & \multirow[t]{2}{*}{1} \\
\hline Female & $585(54.7)$ & 195(55.9) & $390(54.8)$ & & \\
\hline \multicolumn{6}{|l|}{ Cohort age } \\
\hline$<25$ years & $167(15.6)$ & $59(16.6)$ & $108(15.2)$ & \multirow[t]{3}{*}{1.8} & \multirow[t]{3}{*}{0.40} \\
\hline $26-45$ years & $780(73.0)$ & $251(70.5)$ & $529(74.2)$ & & \\
\hline$>45$ years & $122(11.4)$ & $46(12.9)$ & 76(10.7) & & \\
\hline \multicolumn{6}{|l|}{ Address } \\
\hline Maekel & $931(87.1)$ & $308(86.5)$ & $623(87.4)$ & \multirow[t]{2}{*}{0.2} & \multirow[t]{2}{*}{0.7} \\
\hline Outside Maekel & $138(12.9)$ & $48(13.5)$ & $90(12.6)$ & & \\
\hline \multicolumn{6}{|l|}{ Educational level } \\
\hline No formal education & $59(5.5)$ & $21(5.9)$ & $38(5.4)$ & \multirow[t]{3}{*}{0.1} & \multirow[t]{3}{*}{0.93} \\
\hline Primary \& junior & $455(42.6)$ & $152(42.7)$ & $303(42.7)$ & & \\
\hline Secondary \& above & $551(51.5)$ & $183(51.4)$ & $368(51.9)$ & & \\
\hline \multicolumn{6}{|l|}{ Marital status } \\
\hline Married & $505(47.2)$ & $156(43.9)$ & $349(57.1)$ & \multirow[t]{4}{*}{5.2} & \multirow[t]{4}{*}{0.16} \\
\hline Single & $302(28.4)$ & 107(30.1) & 195(31.9) & & \\
\hline Divorced & $98(9.2)$ & $41(11.5)$ & $57(9.3)$ & & \\
\hline Widowed & $107(14.9)$ & $51(14.4)$ & 107(17.5) & & \\
\hline \multicolumn{6}{|l|}{ Employment } \\
\hline Employed & $611(57.2)$ & $201(56.5)$ & $410(57.5)$ & \multirow[t]{2}{*}{0.10} & \multirow[t]{2}{*}{0.79} \\
\hline Unemployed & $458(42.8)$ & $155(43.5)$ & $303(42.5)$ & & \\
\hline \multicolumn{6}{|l|}{ Care entry point } \\
\hline VCT & $620(58.0)$ & $182(51.1)$ & $438(61.5)$ & \multirow[t]{5}{*}{14.5} & \multirow[t]{5}{*}{0.006} \\
\hline In-Patient Wards & $64(6.0)$ & $22(6.2)$ & $42(5.9)$ & & \\
\hline Medical OPD & $255(23.9)$ & $94(26.4)$ & 161(22.6) & & \\
\hline Transfer In & $66(6.2)$ & $32(10)$ & $34(4.8)$ & & \\
\hline Others & $63(5.9)$ & $26(7.3)$ & $37(5.2)$ & & \\
\hline
\end{tabular}


Prior exposure to ART

$\begin{array}{llllll}\text { Yes } & 135(12.6) & 295(82.9) & 629(89.5) & 9.3 & 0.003 \\ \text { No } & 934(87.4) & 61(17.1) & 74(10.5) & & \end{array}$

Baseline $C D 4^{+}$cell count

\begin{tabular}{llllll}
$\leq 200 \mathrm{cells} / \mu \mathrm{l}$ & $621(58.1)$ & $230(65.2)$ & $391(55.2)$ & 9.6 & 0.002 \\
\cline { 1 - 4 }$>200 \mathrm{cells} / \mu \mathrm{l}$ & $440(41.2)$ & $123(34.8)$ & $317(44.8)$ & &
\end{tabular}

Initial WHO clinical stage

Stage 1

$923(87.1) \quad 250(70.6) \quad 673(95.3) \quad 137.8$

$<0.001$

Stage II

$59(5.6) \quad 44(12.4) \quad 15(2.1)$

Stage III

$41(3.9) \quad 25(7.1) \quad 16(2.3)$

Stage IV

37 (3.5)

35(9.9) 2(0.3)

Initial regimen backbone



NNRTI

EFV

$465(43.5) \quad 120(34.1) \quad 345(48.5)$

19.9

$<0.001$

NVP

$598(55.9) \quad 232(65.9) \quad 366(51.5)$

CART changes

Yes

$802(75.0) \quad 40(11.3) \quad 226(31.7)$

52.9

$<0.001$

No

$266(25.0) \quad 315(88.7) \quad 487(68.3)$

Current WHO clinical stage

Stage 1

$982(87.1)$

250(70.6)

673(95.3)

137.8

$<0.001$

Stage II

59 (5.6)

44(12.4) 15(2.1)

Stage III

41 (3.9)

25(7.1)

16(2.3)

Stage IV

37 (3.5)

35(9.9)

2(0.3)

\section{TB Status}


No symptom

$187(17.5) \quad 52(14.6) \quad 135(18.9)$

8.9

0.030

Took IPT

783 (73.2) $\quad 261(73.3) \quad$ 522(73.2)

Took AntiTB

$28(2.6)$

15(4.2)

13(1.8)

Took IPT \& ANTI-TB

$71(6.6)$

28(7.9)

43(6)

Cotrimoxazole Prophylaxis

$\begin{array}{llllll}\text { Yes } & 522(49.4) & 217(61.3) & 305(43.4) & 30.0 & <0.001 \\ \text { No } & 534(50.6) & 137(38.7) & 397(56.6) & & \end{array}$

Missed hospital appointments

$\begin{array}{llllll}\text { Yes } & 603(56.4) & 247(70.8) & 356(50.2) & 40.3 & <0.001 \\ \text { No } & 455(42.6) & 102(29.2) & 353(49.8) & & \end{array}$

Record of Suboptimal Drug adherence

$\begin{array}{llllll}\text { Yes } & 609(57.2) & 248(71.3) & 361(51) & 51.6 & <0.001 \\ \text { No } & 447(42.3) & 100(28.7) & 347((49) & & \end{array}$

\section{Drug Intolerance}

CART

$289(27.0)$

Cotrimoxazole

$23(2.2)$

Anti-TB

$7(0.7)$

\section{Baseline functional status}

Working

$808(75.6) \quad 236(66.5) \quad 572(81)$

27.7

$<0.001$

Ambulatory

$212(19.8)$

Bed-ridden

42 (3.9)

97(27.3) 115(16.3)

Comorbid conditions

Yes

85 (8)

44(12.4) 41(5.8)

14.1

$<0.001$

No

$982(91.9) \quad 312(87.6) \quad$ 670(94.2)

CART changes to similar Analogue

Yes

283 (26.5)

No

786 (73.5)

Weight shift

Positive

889 (83.2) 


\section{Previous Hospitalizations}

\begin{tabular}{|c|c|c|c|c|c|}
\hline Yes & 196(18.6) & $259(50)$ & $108(15.2)$ & 30.22 & $<0.001$ \\
\hline No & $860(81.4)$ & $259(50)$ & $601(84.8)$ & & \\
\hline
\end{tabular}

Abbreviations: TDF, Tenofovir disoproxil fumarate; FTC: Emtricitabine; AZT: Zidovudine (AZT); 3TC: Lamivudine; ABC: Abacavir; D4T: Stavudine; NVP: Nevirapine; EFV: Efavirenz.

OPD: Outpatient department; VCT: Voluntary Counseling and Testing center

SMT TMP: Sulfamethoxazole Trimethoprim.

ART, antiretroviral therapy; Cases, plasma HIV RNA >1000 copies/mL; Control: HIV RNA $\otimes 1000$ copies/mL);

NNRTI, non-nucleoside reverse transcriptase inhibitor; NRTI, nucleoside reverse transcriptase inhibitor

\section{Demographic and Clinical characteristics of patients}

Among the 1068 participants (356 cases and 712 controls), females account for $45.2 \%(\mathrm{~N}=483)$ and median age at enrollment was 46 years (IQR: 39-51 years) ranging from 15 years to 86 years. Males were older at enrollment than females (39 years [IQR: 33-43] vs 32 years [IQR: $27-37]$ ), p-value< 0.001 . The mode of entry to clinical care for cases was less through VCT than controls $(51.1 \%$ vs $61.5 \%, p<0.001)$ (Table1). The median time to cART failure was 37 months (IQR: 24 months -47 months). At baseline, participants had a median CD $4^{+}$cell count of 170 cells/ $\mu \mathrm{l}$ (IQR $\left.=84.5-275\right)$ (males: $150.5 \mathrm{cells} / \mu \mathrm{l}$ (IQR: 68-246) vs females: 187 cells/ $\mu$ l (103-297) and advanced HIV (CD4 ${ }^{+}$cell count $<200$ cells/ul) was present in $621(58.1 \%)$. The frequency of late presentation (LP) was 919 (87.0\%). In the period before 2005, after 2005-2010, >2010-2014, and >2014-2020, the frequencies were 82 (91.1\%), 453 (92.6\%), 281 (85.4\%), and $103(69.6 \%)$, respectively. Among the LPs, 303 (28.3\%) presented very late (CD4+ cell count $201-350$ cells/ $\mu$ l or WHO clinical stage III), and 621(58.1\%) presented extremely late (CD4+ cell count $\leq 200 \mathrm{cells} / \mu \mathrm{l}$ and/or WHO clinical stage IV) for HIV care.

\section{First-line ART treatment-related condition}

Median duration on first line regimen was 44 months (IQR: 20 months - 76 months). The most frequently used first-line cART backbones were: d4T+3TC in 39.8\% $(n=425)$ followed by; AZT-3TC, 35\% $(n=374)$ and TDF+FTC, 22.4\% ( $n=239)$. Others were TDF+3TC, 1.6\% $(n=17)$ and ABC+3TC, 1.2\% $(n=13)$. Majority of the cases were initially placed on d4T $+3 \mathrm{TC}(49 \%)$ and most of the controls were started on AZT + 3TC (36.7\%), $p<0.001$.

Among the controls, 124(11.9\%) had viremia (plasma HIV-RNA $\geq 50-999$ copies/mL [9] at the most recent visit), and $553(78.1 \%$ ) had viral suppression ( $<50$ copies $/ \mathrm{mL}$ ) [10] in the most recent VL test. Among 
cases, the median viral load was 39,810 copies/mL (6,615.50 copies/mL - 195,199 copies/mL).

Further, $75 \%$ of the study subjects had a history of cART changes with $26.5 \%$ changing to similar analogs. The cART changes were mostly prompted by adverse drug reactions/toxicity, 297 (27.8\%) and drug stock-outs, 280 (25.6\%). The median interval between HIV diagnosis and cART initiation was 3 months (IQR: 1-9) before 2005. Between 2005 and 2010 it was 2 months (IQR: 0.00-14), and it was 5 months (IQR: 0.00-45.5) between 2010 and 2016.As of 2016 though, the time to initiation is 1 month (IQR: 0.00-53). Regarding opportunistic infections and events among the study participants the most frequent was weight loss $(n=138)$, followed by recurrent pneumonia $(n=103)$, chronic cough $(n=95)$, oral/vaginal thrush $(n=56)$, herpes zoster $(n=53)$, chronic fever $(n=50)$ and others $(n=94)$. Among the study participants, 9.3\% were treated with anti-TB during their follow-up while $73.2 \%$ and $50 \%$ of the participants had received Isoniazid and Cotrimoxazole prophylaxis respectively. See figure 2 and Table 1 for additional information.

\section{Table 2. Multivariate analysis of sociodemographic predictors of treatment failure}




\begin{tabular}{|lllll|}
\hline Variables & \multicolumn{2}{l}{ Case vs Controls } & & \\
\cline { 2 - 5 } & COR $(95 \% \mathrm{Cl})$ & P value & aOR (95\% Cl) & P value \\
\hline Address & & & & \\
\hline Maekel & 1 & & - & - \\
\hline Outside Maekel & $1.08(0.74-1.57)$ & 0.69 & - & - \\
\hline Occupation & & & - & - \\
\hline Employed & 1 & & - & - \\
\hline Unemployed & $1.04(0.87-1.35)$ & 0.75 & - & - \\
\hline Marital status & & & - & - \\
\hline Married & 1 & & - & - \\
\hline Single & $1.22(0.91-1.66)$ & 0.183 & - & - \\
\hline Divorced & $1.6(1.03-2.5)$ & 0.036 & - & - \\
\hline Widow (ed) & $1.1(0.73-1.56)$ & 0.74 & - & - \\
\hline Educational level & & & - & - \\
\hline No formal education & 1 & & - & - \\
\hline Primary & $0.85(0.46-1.57)$ & 0.61 & - & - \\
\hline Junior & $0.96(0.53-1.73)$ & 0.88 & - & - \\
\hline Secondary & $0.89(0.5-1.56)$ & 0.67 & - & - \\
\hline Higher level & $1.02(0.49-2.12)$ & 0.97 & - & - \\
\hline
\end{tabular}

Table 3. Multivariable analysis of Clinical predictors of treatment failure 


\begin{tabular}{|c|c|c|c|c|}
\hline \multirow[t]{2}{*}{ Variables } & \multicolumn{4}{|c|}{ Case vs Controls } \\
\hline & $\operatorname{cOR}(95 \% \mathrm{Cl})$ & $\begin{array}{l}P \\
\text { value }\end{array}$ & aOR $(95 \% \mathrm{Cl})$ & $\begin{array}{l}P \\
\text { value }\end{array}$ \\
\hline \multicolumn{5}{|l|}{ NRTI } \\
\hline TDF+FTC & 1 & $<0.001$ & 1 & $<0.001$ \\
\hline $\mathrm{AZT}+3 \mathrm{TC}$ & $\begin{array}{l}2.41(1.40- \\
4.16)\end{array}$ & 0.002 & $\begin{array}{l}2.70(1.65- \\
4.41)\end{array}$ & $<0.001$ \\
\hline$A B C+3 T C$ & $\begin{array}{l}4.38(1.10- \\
17.51)\end{array}$ & 0.037 & $\begin{array}{l}4.73(1.18- \\
18.92)\end{array}$ & 0.028 \\
\hline D4T+3TC & $\begin{array}{l}4.07(2.2 .12- \\
7.79)\end{array}$ & $<0.001$ & $\begin{array}{l}5.00(3.03- \\
8.20)\end{array}$ & $<0.001$ \\
\hline \multicolumn{5}{|c|}{ Prior Exposure to ART } \\
\hline No & 1 & \multirow[t]{2}{*}{0.115} & 1 & \multirow[t]{2}{*}{0.002} \\
\hline Yes & $\begin{array}{l}1.78(0.87- \\
3.64)\end{array}$ & & $\begin{array}{l}2.28(1.35- \\
3.86)\end{array}$ & \\
\hline \multicolumn{5}{|c|}{ Care entry point } \\
\hline VCT & 1 & \multicolumn{3}{|l|}{0.426} \\
\hline In patient & $\begin{array}{l}0.749(0.38- \\
1.48)\end{array}$ & \multicolumn{3}{|l|}{0.749} \\
\hline Medical OPD & $\begin{array}{l}1.11(0.76- \\
1.61)\end{array}$ & \multicolumn{3}{|l|}{0.380} \\
\hline Others & $\begin{array}{l}1.61(0.81- \\
3.18)\end{array}$ & \multicolumn{3}{|l|}{0.155} \\
\hline Transfer in & $\begin{array}{l}1.62(0.66- \\
3.95)\end{array}$ & \multicolumn{3}{|l|}{0.291} \\
\hline \multicolumn{5}{|l|}{ NNRTI } \\
\hline EFV & 1 & \multirow[t]{2}{*}{0.310} & & \\
\hline NVP & $\begin{array}{l}1.25(0.81- \\
1.94)\end{array}$ & & & \\
\hline \multicolumn{5}{|c|}{ Record of Sub-optimal Drug adherence } \\
\hline No & 1 & \multirow[t]{2}{*}{$<0.001$} & 1 & \multirow[t]{2}{*}{$<0.001$} \\
\hline Yes & $\begin{array}{l}3.06(2.20- \\
4.26)\end{array}$ & & $\begin{array}{l}3.08(2.22- \\
4.28)\end{array}$ & \\
\hline \multicolumn{5}{|c|}{ Baseline functional Status } \\
\hline Working & 1 & 0.010 & 1 & 0.010 \\
\hline
\end{tabular}


Ambulatory/Bed-ridden

1.63(1.12-

2.37)

$1.61(1.12-$

2.31)

\section{Any Comorbidities}

\begin{tabular}{|c|c|c|c|c|}
\hline No & 1 & \multirow[t]{2}{*}{0.001} & 1 & \multirow[t]{2}{*}{0.002} \\
\hline Yes & $\begin{array}{l}2.55(1.46- \\
4.45)\end{array}$ & & $\begin{array}{l}2.37(1.36- \\
4.10)\end{array}$ & \\
\hline \multicolumn{5}{|l|}{ Duration of cART } \\
\hline$>5$ years & 1 & \multirow[t]{2}{*}{$<0.001$} & 1 & \multirow[t]{2}{*}{$<0.001$} \\
\hline$<5$ years & $\begin{array}{l}5.93(3.95- \\
8.90)\end{array}$ & & $\begin{array}{l}5.90(3.95- \\
8.73)\end{array}$ & \\
\hline \multicolumn{5}{|l|}{ SMX TMP } \\
\hline No & 1 & \multirow[t]{2}{*}{$<0.001$} & 1 & \multirow[t]{2}{*}{$<0.001$} \\
\hline Yes & $\begin{array}{l}2.01(1.44- \\
2.81)\end{array}$ & & $\begin{array}{l}2.00(1.44- \\
2.78)\end{array}$ & \\
\hline \multicolumn{5}{|l|}{ Baseline CD4+ cell count } \\
\hline$<50$ cells/ $\mu \mathrm{l}$ & 1 & 0.039 & 1 & 0.039 \\
\hline$>50-100$ cells $/ \mu \mathrm{l}$ & $\begin{array}{l}1.91(1.11- \\
3.26)\end{array}$ & 0.019 & $\begin{array}{l}1.92(1.12- \\
3.28)\end{array}$ & 0.017 \\
\hline$>100-200$ cells $/ \mu \mathrm{l}$ & $\begin{array}{l}1.05(0.63- \\
1.72)\end{array}$ & 0.974 & $\begin{array}{l}1.07(0.65- \\
1.76)\end{array}$ & 0.787 \\
\hline$>200-350$ cells/ $\mu \mathrm{l}$ & $\begin{array}{l}1.55(0.91- \\
2.20)\end{array}$ & 0.305 & $\begin{array}{l}1.57(0.929- \\
2.66)\end{array}$ & 0.092 \\
\hline$>350$ cells $/ \mu \mathrm{l}$ & $\begin{array}{l}1.13(0.58- \\
2.20)\end{array}$ & 0.992 & $\begin{array}{l}1.12(0.57- \\
2.18)\end{array}$ & 0.745 \\
\hline $\begin{array}{l}\text { The duration between diagnosis and } \\
\text { initiation of CART }\end{array}$ & $\begin{array}{l}0.992(0.986- \\
1.00)\end{array}$ & 0.007 & $\begin{array}{l}0.992(0.986- \\
1.00)\end{array}$ & 0.006 \\
\hline
\end{tabular}

Abbreviations: TDF, Tenofovir disoproxil fumarate; FTC: Emtricitabine; AZT: Zidovudine (AZT); 3TC: Lamivudine; ABC: Abacavir; D4T: Stavudine; NVP: Nevirapine; EFV: Efavirenz.

OPD: Outpatient department; VCT: Voluntary Counseling and Testing center

SMT TMP: Sulfamethoxazole Trimethoprim.

Cl: Confidence interval

ART, antiretroviral therapy; Cases, plasma HIV RNA >1000 copies/mL; Control: HIV RNA $\otimes 1000$ copies $/ \mathrm{mL})$; 
NNRTI, non-nucleoside reverse transcriptase inhibitor; NRTI, nucleoside reverse transcriptase inhibitor

\section{Multivariable analysis of predictors of first-line cART treatment failure}

Multivariable logistic regression analysis was then performed, higher odds of failure were observed among patients who were initiated on initial cART regimen NRTI of D4T+3TC were five times higher odds to experience TF (aOR=5; 95\% Cl: 3.03-8.2, p-value $<0.001)$ while those initiated on ABC+3TC showed similar odds of failure (aOR=4.7; 95\% Cl: $1.18-18.82$, $\mathrm{p}$-value=0.028). Moreover; Patients initiated on initial regimen backbone $A B C+3 T C$ were also found to be three times as likely to fail as compared to those on TDF+FTC (aOR=2.7 95\% Cl: 1.65-4.41, p-value<0.001). In addition, patients who had prior exposure to ART were nearly three times as likely to fail first-line treatment as compared to those without $(\mathrm{aOR}=2.28$; $95 \% \mathrm{Cl}: 1.35-3.86)$. Record of sub-optimal adherence was also found to be a risk factor for TF with such patients having a three-fold higher odds of $\mathrm{TF}(\mathrm{aOR}=3.08 ; 95 \% \mathrm{Cl}$ : 2.22-4.28). Baseline functional status was also a predictor of TF as patients who were ambulatory /bedridden at presentation to care being 1.5 times as likely to fail as compared to those who were functional ( $\mathrm{aOR}=1.61 ; 95 \% \mathrm{Cl}: 1.12-4.28)$. Study participants who had comorbid clinical conditions were also at higher risk of TF; i.e. they were twice higher odds to fail as compared to their counterparts ( $\mathrm{aOR}=2.37 ; 95 \% \mathrm{Cl}: 1.36-4.10)$. Another important finding is the fact that shorter duration on treatment was associated with higher odds of TF with participants who were on CART for less than five years being 6 times more likely to fail than those who were on treatment for longer $(\mathrm{aOR}=5.90 ; 95 \% \mathrm{Cl}$ : 3.95-8.73). Meanwhile; patients who took/are taking $\mathrm{SMX} / \mathrm{TMP}$ were found to be twice as likely to experience TF $(\mathrm{aOR}=2.0,95 \% \mathrm{Cl}, 1.44-2.78)$. Baseline immune-status (CD4+ cell count) was also a predictor of TF, with those lower $\mathrm{CD} 4^{+}$cell having lesser odds of failure than higher $\mathrm{CD} 4^{+}$, see Table 3 .

A separate multivariable logistic regression was also conducted to evaluate for sociodemographic determinants of TF. Although in univariate analysis participants who were divorced showed 1.6 times higher odds of TF compared to those married, none of the variables remained significant in multivariate analysis. (Table 2)

\section{Longitudinal changes in absolute CD4+ cell count}

The median CD4+ cell count at baseline was significantly lower for cases than controls $(150.0 \mathrm{cells} / \mu \mathrm{l}$; IQR $=70.5-259.0)$ vs (181.5 cells/ $\mu l ; I Q R=95.3-286.0), p<0.001$ (Mann Whitney-U test). After 6 months of treatment, both cases and controls showed similar immune recoveries where median CD4+ cell count for cases showed a marked response to $258 \mathrm{cells} / \mu \mathrm{l}(\mathrm{IQR}=148.0-367.0)$ almost catching up to that of controls which amounted to 264 cells $/ \mu \mathrm{I}(\mathrm{IQR}=150.3-375.5), \mathrm{p}=0.787$. After a year of treatment however, the median CD4+ cell count among cases declined to 255 cells/ $\mu \mathrm{l}(\mathrm{IQR}=148.5-369.5)$ while it increased to $290.5 \mathrm{cells} / \mu \mathrm{l}(\mathrm{IQR}=187.00-406.25)$ among controls, this was statistically significant $(p=0.012)$. Moreover; two years following treatment with cART the difference became even more pronounced, with the median count for cases only increasing to $276.0 \mathrm{cells} / \mu \mathrm{l}(\mathrm{IQR}=179.8-445.0)$ in contrast median CD4+ cell count for controls showed marked recovery as it mounted to 337 cells/ $\mu \mathrm{l}(\mathrm{IQR}=220.0-480.3)$, 
which is a statistically significant difference $(p=0.001)$. In the exact same manner 3 years following treatment a similar pattern in immune response to treatment was observed, cases had a median count of 287.0 cells $/ \mu \mathrm{l}(\mathrm{IQR}=186.5$ - 425.0) whereas controls had around 379.0 cells $/ \mu \mathrm{L}(\mathrm{IQR}=259.0-515.5)$, with a $\mathrm{p}$ value $<0.001$. (Figure 2 )

\section{CD4 ${ }^{+}$cell count Kinetics}

The median $\mathrm{CD} 4^{+}$cell count recovery rate in the first six months of treatment was higher among cases as compared to controls ( $21.6 \mathrm{Vs} 17.6 \mathrm{cell} / \mathrm{s} / \mathrm{l} / \mathrm{month}$ ) indicating that cases have a good recovery rates following treatment initiation even better than that of controls, though not statistically significant ( $p$ value $=0.34$, Mann Whitney $-U$ test). However; The rate of $C D 4^{+}$cell count increment in the 6 to 12 month interval was 1.3 cells/ $\mu \mathrm{l} /$ month (IQR: $-5.8-10.5$ ) and 5 cells/ $\mu \mathrm{l} /$ month (IQR: 28.8, -2-12.5) for controls and cases, respectively $(p=0.058)$. In similar fashion, in the third interval (12 months to 24 months) the median rates of $\mathrm{CD} 4^{+}$cell count increments were significantly lower for cases than control $(2.3 \mathrm{cells} / \mu \mathrm{l}$ /month $(-4.6-8.3)$ vs 3.1 cells/ $\mu \mathrm{l} /$ month $(-2-8.4)$, p value $=0.034)$. Moreover; significant differences were observed in the median rate of CD4 + cell count increments in the 24-36 months of treatment with cases showing minimal increment as compared to their control counterparts (cases: 0.7 cells/ $\mu \mathrm{l} / \mathrm{month}(-4.5-$ 6.4) vs controls: 3.1 cells/ $\mu \mathrm{l} /$ month $(-2-8.4), p=0.003)$. See figure 3 and Table 4.

The table below the graph indicates the median rate of $\mathrm{CD} 4^{+}$cell count increase (cells/ $\mu \mathrm{l} / \mathrm{month}$ ) between the different periods and their IQR.

Table 4: Median CD4 ${ }^{+}$cell count in Cells/ $\mu \mathrm{l} /$ month (IQR) change per month during the first 3 years of treatment initiation

\begin{tabular}{|lllll|}
\hline $\begin{array}{l}\text { Median Rate in cells/ } \\
\mu \text { /month (IQR) }\end{array}$ & $\begin{array}{l}\text { Rate 1: } \\
\text { Baseline-6 } \\
\text { Months }\end{array}$ & $\begin{array}{l}\text { Rate 2: } \\
\text { >6 Months-12 } \\
\text { Months }\end{array}$ & $\begin{array}{l}\text { Rate 3: } \\
\text { >12 Months -24 } \\
\text { Months }\end{array}$ & $\begin{array}{l}\text { Rate 4: } \\
\text { 24 Months -36 } \\
\text { months }\end{array}$ \\
\hline Case & $\begin{array}{l}21.6(4.4- \\
40.2)\end{array}$ & $1.3(-5.8-10.5)$ & $2.3(-4.6-8.3)$ & $0.7(-4.5-6.4)$ \\
\hline Control & $\begin{array}{l}17.6(4.7- \\
32.3)\end{array}$ & $5(-2-12.5)$ & $4(-0.5-10.4)$ & $3.1(-2-8.4)$ \\
\hline *P value & 0.34 & $\mathbf{0 . 0 5 8}$ & $\mathbf{0 . 0 3 4}$ & $\mathbf{0 . 0 0 3}$ \\
\hline
\end{tabular}

* Mann Whitney-U test

\section{Discussion}

This retrospective analysis of data from multiple HIV/AIDs treatment sites is not unique in exploring the factors associated with TF in patients from Asmara, Eritrea. The study discovers a catalogue of TF risk factors in addition the study also highlights that cases had a profoundly lower immune response to 
treatment and significantly shorter duration on CART in contrast to controls. Specifically, the data suggests that TDF+EFV-based-CART appeared more effective than non-TDF-based regimens (AZT+3TC, $A B C+3 T C$, and $D 4 T+3 T C$ ). In general, these findings align with previous studies which suggested that DRMs to TDF are limited [16] and that its safety record is relatively good [17]. Importantly, reports suggest that the efficacy of AZT, D4T, and ABC-based cART has been compromised by the emergence of DRMs in the region [16]. In addition, studies on NVP-based cART vs EFV-based ART have suggested that the former is marginally less efficacious [18]. However, our analysis did not uncover such associations.

Multiple explanations can be invoked to explain why TDF+EFV-based CART is less associated with TF. First, adverse effects (vomiting, diarrhea, among others) associated with AZT, ABC, and D4T may potentially compromise treatment or account for differences in adherence rates. Importantly, a study in Kenya demonstrated that TDF had a lower drug change rate (1.9 per 100 person-years) compared to D4T (27 per 100 person-years) [19] - the changes were largely attributed to adverse drug reactions (mostly lipodystrophy and polyneuropathy). Interestingly, adverse drug reactions were a prominent reason for changes in initial CART in this setting. Another possibility with far-reaching implications is the presence of resistance mutations (RMs). Incredibly, research suggests that $\geq 50$ - $90 \%$ of patients experiencing virologic failure on first-line cART with viral count > 1000 copies/ml have NNRTI resistance [20,21]. In particular, patients in SSA with first-line TF to NNRTI + NRTIs often present with the M184V mutation (associated with NRTIs - 3TC/FTC) and the K103N mutation (NNRTIs) [22,23]. Resistance testing is not part of the treatment protocol in Eritrea as drug resistance survey (DRS) conducted in 2017 revealed RM less than $10 \%$ among newly diagnosed, which is less than the WHO threshold for routine resistance assay.

However, indirect evidence of the potential role of DRMs was noted in this study. First, large proportions of patients $(77.4 \%)$ were on 3TC-based regimens and sub-optimal response to treatment in patients who were switched to 3TC-based regimens was also noted. More importantly, patients were placed on failing regimens for an extended duration of time. Petersen et al. demonstrated that delayed second-line ART switch has been associated with the emergence of DRMs [24]. A different line of evidence that points to the possible presence of DRMs was the observed relationship between prior antiretroviral drug use (PAU) and VF. In general, prior exposure to ART - regardless of viral load count - has been linked to an increased likelihood of VF [22]. Others have also suggested that the increased risk of TF observed in patients with PAU is largely attributable to pre-treatment drug resistance (PDR) [25]. According to this report, HIV-infected adults in SSA starting first-line NNRTI-based cART and have a history of PAU, i.e. ART or single dose Nevirapine (sdNVP) for PMTCT, were more likely to have VF [25]. In addition, Cutrell and Jodlowski described that in the absence of resistance testing, it's prudent to assume resistance to drug regimens with a relatively low genetic barriers to resistance, such as EFV, 3TC, FTC, Raltegravir, or Elvitegravir, if these agents were part of a previously failing regimen [9]. Therefore, the use of differentiated, non-NNRTI-based empiric first-line therapy has been recommended in such patients. However, this is impractical in Eritrea due to the limited range of ART available. Regardless, the extensive use of 3TC in patients with poor response to initial CART or with PAU should be reconsidered. 
Furthermore, similar to other studies in the region [26, 27], we noted that sub-optimal adherence to cART was a principal contributor to VF. Sub-optimal adherence can lead to high healthcare costs, poor patients outcomes (increased HIV-related morbidity and mortality), the emergence of DRMs, and increased community HIV transmission [9]. Numerous studies have demonstrated that even in high-functioning health systems, adherence to cART remains a major obstacle in HIV/AIDS treatment programs [9]. For instance, a recent study in Ethiopia suggested that the likelihood of VF was 5.4 fold higher among those who had poor adherence [28]. Likewise, poor adherence to cART as a correlate of VF was reported by investigators in Kenya [29] and Ethiopia [26,27]. In summary, we can maintain that despite the relative heterogeneity of study designs and the diverse nature of backgrounds studied in SSA; inadequate adherence to CART is a ubiquitous contributor to TF in the region. Multiple socio-demographic, environmental, and behavioral factors are known to influence sub-optimal adherence. These include older age, living conditions/situation, stigma, early-stage HIV infection, comorbid mental health conditions, DRMs, adverse drug effects, DDIs, poor tolerability, polypharmacy drug stock-outs and substance use [9]. To understand and address the challenges associated with sub-optimal cART adherence, a better appreciation of the relevant determinants is required. Unfortunately, it can be argued that while most studies in the region highlight the importance of sub-optimal adherence to CART; its determinants are poorly described[30]. In this regard, the study corroborates the findings of a recent meta-analysis which identified toxicity as a prominent cause of poor adherence, 58\% (95\% Cl: 46, 69\%; Range: $14.4-$ 88.5\%) [31]. Beyond these issues, concerns regarding the diagnostic accuracy of self-reported adherence data have been highlighted [32]. That being said, the inability to obtain a reliable quantification of the adherence process over time, can thus be a barrier to intervention. These concerns are highly relevant in this setting.

By most accounts, the problem of poor adherence to CART in treatment programs in SSA is formidable. However, success has been demonstrated for mitigation efforts that prioritize the integration of adherence interventions as part of routine clinical care. In the United States, cART adherence is discussed at every visit, and patients triaged as poor adherers are promptly referred for counseling or enhanced adherence intervention or support [20]. Alternatively, some authors support the idea that chronic non-cART adherers should be placed on regimens with a higher barrier to resistance - boosted protease inhibitors (PI) or Dolutegravir (DTG) [9]. In SSA, the latter option will require expansion of cART choices. This has important cost implications which severely limit its practicality in settings like Eritrea.

Further, we demonstrated that low $\mathrm{CD} 4^{+}$cell count (baseline CD $4^{+}$cell count of $<50$ and $\leq 100$ cells/ $\mu$ l) were associated with increased odds of VF. Comparable results have been reported in Ethiopia [26,3335], and Kenya [36], among others. To explain this relationship, the inverse relationship between $\mathrm{CD} 4^{+}$cell count and viral replication at specific stages of the disease has been invoked [33]. In addition, a low CD4 ${ }^{+}$ cell count is a marker of advanced disease, hence the potential presence of HIV-defining infections. In important respects, the foregoing discussion underscores the fact that delayed/late diagnosis/presentation (919 (87.0\%) presented late) is one of the biggest problems facing HIV treatment programs in Asmara, Eritrea. Admittedly, problems related to study design may undermine the accuracy 
of the late presentation estimates. Either way, we believe that our estimates are largely reliable. Apart from $\mathrm{CD} 4^{+}$cell count, surrogate pointers to the late presentation as a major problem in this setting can be gleaned from several associations in the bivariate analysis. These include the proportion of patients with WHO Stage III and IV disease at baseline, baseline functional status, comorbidities, and the inverse relationship between time of HIV diagnosis and initiation of cART. Predictably, some of these factors emerged as predictors of VF in the multivariable model. Overall, we can conclude that the observed gap between HIV diagnosis and treatment for some patients requires particular scrutiny since it can compound the problem of late presentation.

Previous studies have shown that late entry to care is harmful in multiple ways - worse prognosis, shorter survival, and less benefit from cART [37,38]. Documented factors associated with late presentation include male gender, older age, stigma, poor mental health [38], low-risk perception, discrimination, lack of spousal HIV status disclosure, lower-income, poor social support, level of education, lack of awareness about the need for early HIV, access to testing and treatment sites, limited investments in community, and structural interventions [37 - 44]. Thus, drivers of late presentation are from diverse domains (economic, social, demographic, geographic, and psychosocial) and are undeniably complex and contextualized. As such, no two jurisdictions share the same complement of factors. This argument underscores the importance of local data. Unfortunately, the factors associated with late presentation are under-described in Eritrea. Thus, efforts to improve early HIV diagnosis (e.g. mobile- and home-based testing and counseling), early linkage to chronic HIV care centers, and timely initiation to cART should be prioritized. Formulation of new treatment models for late presenters should also be addressed (particularly $\mathrm{CD} 4^{+}$cell count $\leq 200$ cells $/ \mu \mathrm{l}$ and/or WHO clinical stage III and IV). There is strong evidence from the region indicating that intervention models mandating weekly or bi-weekly contact with care sites can work. These models are generally credited with early identification and treatment of opportunistic infections (OIs) and reductions in morbidity and mortality.

To further understand the relationship between treatment and immunological response. We considered it important to evaluate the kinetics of $\mathrm{CD} 4^{+}$cell count at specified intervals. In the process, we noted some outstanding points. Immunological recovery (> 500 cells $/ \mathrm{mm}^{3}$ ) was generally poor in a majority of patients (cases: $14 \%$ vs Control: $28 \%$ ), who achieved it after 36 months of treatment. This was contrary to the conclusions in a recent review which noted that achievement of sustained virologic suppression with CART is typically associated with a steady increase in peripheral blood CD $4^{+}$cell count recovery $(>500$ cells/ $\mu$ l) [9]. They also noted that $\sim 15-20 \%$ of patients, particularly late presenters who start therapy at $\mathrm{CD} 4^{+}$cell count $\left(<200\right.$ cells $/ \mu \mathrm{l}$ ) will plateau at a $\mathrm{CD} 4^{+}$cell count below the immunological recovery threshold. More importantly, we also demonstrated that the increment rate of $\mathrm{CD} 4^{+}$cells/ $\mu \mathrm{l} /$ month differed significantly between cases and controls - a finding that is by no means unique [42]. However, the overall $\mathrm{CD} 4^{+}$cells/ $\mu \mathrm{l} /$ month was substantially less than what is recommended by some investigators/or guidelines. For instance, some studies concluded that CD $4^{+}$cell count gains $<100$ cells/ $\mu \mathrm{l} /$ year can be used to identify patients at risk of hard endpoints such as AIDS, serious non-AIDS events, and death [42]. 
Finally, we have to note that our model suggests that Cotrimoxazole prophylaxis (CPT) was a predictor of virologic failure. This finding counteract with results from a study conducted in Ethiopia [43]. In their opinion, the relationship was potentially linked to the fact that CPT boosts the immune status of patients in that CPT directly prevents opportunistic infections, and leads to the reduction in the incidence of virologic failure associated with different causes. However, in this jurisdiction, we think that the most plausible explanation relates to the fact that patients experiencing VF are more likely to present with Ols and are thus more likely to be placed on Cotrimoxazole prophylaxis by clinicians. Therefore, the finding merely raises questions regarding triggers for Cotrimoxazole prophylaxis.

\section{Strengths and limitations of the Study}

To the best of our knowledge, our study is the first to evaluate the factors associated with virologic failure in Eritrea. Regardless, it has several limitations. First, the study uses secondary data collected retrospectively. This approach has been associated with the incompleteness of clinical data. Moreover, underreporting/missing data elements can lead to biases - particularly if they are systemic. Secondly, the contribution of HIV drug resistance to VF was not assessed. Lastly, adherence information was largely based on self-report. However, recall and social desirability bias may undermine the reliability of this approach. Despite these limitations, we would like to highlight some strengths: first, information on a large number of variables including demographic information; care entry point; prior exposure to cART; date of HIV diagnosis; date of cART initiation and subsequent treatment history; and clinical outcomes were collected. The availability of this information permitted an in-depth analysis of multiple secondary objectives. Second, the sample size was fairly large for multiple variables thereby strengthening the robustness of our results.

\section{Conclusion}

This multi-center analysis demonstrates that the HIV/AIDS treatment program in Asmara, Eritrea requires optimization in multiple domains. Unlike other countries in the region, patients in this jurisdiction are older. At baseline, majority of the participants presented with advanced HIV, thereby making late presentation a major problem with important individual and public health consequences. Further, it can be asserted that although viral suppression was achieved in a significant number of cases, immunological recovery was poor. Multivariate analysis showed there were multiple factors associated with increased likelihood of VF. Looking into the future, we believe that there is a need for additional resources and efforts targeted at the optimization of CART adherence, diversification of CART regimens, and interventions directed at enhancing early HIV diagnosis and prompt initiations of treatment. Robust evaluation and monitoring in the first years following treatment initiation will be paramount to detecting poor treatment response and subsequent action, including surveillance of DRMs are needed.

\section{Declarations}

\section{Ethical approval and consent to participate}


Ethical approval for this study was obtained from the Eritrean Ministry of Health (MOH) Research Ethical Committee. Information on the maintenance of data confidentiality and integrity was also provided. As the study is based on patients' clinical card records, consent for the data access was waived by the ethical committee in place of the patients. This study conforms to the principles outlined in the Declaration of Helsinki.

\section{Consent for publication}

Consent for publication is also waived by the ethical committee in place of patients as the data is secondary.

\section{Availability of data and materials}

The dataset supporting the conclusions of this article are available from the corresponding author on reasonable request.

\section{Conflict of Interest}

The authors have no conflict of interest to declare in this study.

\section{Financial support}

Data was collected by ART focal personnel with incentive and material support obtained from the Eritrean Ministry of Health, CDC division.

\section{Authors' contributions}

Conceptualization: Samuel Tekle Mengistu

Data curation: Samuel Tekle Mengistu, Ghirmay Ghebrekidan, Hermon Berhe Ghebrat, Nahom Asmerom, Amon Solomon Ghebrenegus, Filimon Gebretsadik, and Naod Yeibyo

Formal analysis: Samuel Tekle Mengistu, Hermon Berhe Ghebrat, Araya Berhane Mesfn, and Negassi Leake

Investigation: Samuel Tekle Mengistu, Oliver Achila, Ghirmay Ghebrekidan Ghebremeskel, and Amanuel Kidane

Methodology: Samuel Tekle Mengistu, Hermon Berhe Ghebrat, Araya Berhane Mesfn, and Negassi Leake Project administration: Samuel Tekle Mengistu, Hermon Berhe Ghebrat, Ghirmay Ghebrekidan, and Nahom Asmerom

Writing - original draft: Samuel Tekle Mengistu, Oliver Achila, Ghirmay Ghebrekidan Ghebremeskel, Filimon Gebretsadik, and Nahom Asmerom 
Writing - review\& editing: Samuel Tekle Mengistu, Ghirmay Ghebrekidan Ghebremeskel, Oliver Achila, Amanuel Kidane Hermon Berhe, Filimon Gebretsadik, and Amon Solomon.

\section{Acknowledgment}

The authors would like to thank the clinical staff who supported this work at Orotta National Referral Hospital, Halibet National Referral Hospital, Haz Haz Zonal Referral hospital and Sembel Hospital. We are grateful to National Communicable Disease Control Division, Eritrean Ministry of Health, and ART Health Management Information System (HMIS) developers and technicians. Our sincere appreciation goes to Dr. Fanus Yemane, Dr. Simon Tesfay, Dr. Yonathan Tesfalidet, and Mr. Thomas Asfaha.

\section{References}

1. UNAIDS. Global HIV \& AIDS statistics - 2021 fact sheet. http://www.unaids.org/en/resources/factsheet. 2021.

2. Das M, Chu PL, Santos G-M, Scheer S, Vittinghoff E et al. Decreases in Community Viral Load Are Accompanied by Reductions in New HIV Infections in San Francisco. PLoS One. 2010;5:e11068.

3. WHO. Consolidated Guidelines on the Use of Antiretroviral Drugs for Treating and Preventing HIV Infection: Recommendations for a Public Health Approach. 2nd. Geneva; 2016.

4. GBD 2019 Diseases and Injuries Collaborators. Global burden of 369 diseases and injuries in 204 countries and territories, 1990-2019: a systematic analysis for the Global Burden of Disease Study 2019. Lancet. 2020;396:1204-22.

5. Iwuji CC, Shahmanesh M, Koole O, Herbst K, Pillay D, Siedner MJ BK behalf of the H-DN. Clinical outcomes after first-line HIV treatment failure in South Africa: the next cascade of care. HIV Med. 2020;21:457-62.

6. WHO. Tackling HIV Drug Resistance: Trends, Guidelines And Global Action. 2017.

7. Kantor R, Diero L, DeLong A, Kamle L, Muyonga S, Mambo F WE et al. Misclassification of First-Line Antiretroviral Treatment Failure Based on Immunological Monitoring of HIV Infection in ResourceLimited Settings. Clin Infect Dis. 2009;49:454-62.

8. Waruru A, Muttai H, Ng'ang'a L, Ackers M, Kim A, Miruka F et al. Positive Predictive Value of the WHO Clinical and Immunologic Criteria to Predict Viral Load Failure among Adults on First, or Second-Line Antiretroviral Therapy in Kenya. PLoS One. 2016;11:e0158881.

9. Cutrell J, Jodlowski T BR. The management of treatment-experienced HIV patients (including virologic failure and switches). 2020;7:1-15.

10. WHO. World Health Organization. Consolidated guidelines on HIV prevention, diagnosis, treatment and care for key populations 2016 update. World Health Organization [Internet]. 2016. Available from: https://apps.who.int/iris/bitstream/handle/10665/246200/9789241511124\%0Aeng.pdf;jsessionid=CE7EDD654B0B5FCEB1A736F0457485B8? sequence $=1$

11. AD L. Genetic barriers to resistance and impact on clinical response. Med Gen Med. 2005;7:69. 
12. Disease C, Division C, Health MOF. Eritrean antiretroviraltherapy guidelines 2021. 2021.

13. Rohr JK, Ive P, Horsburgh CR, Berhanu R, Shearer K MM et al. Developing a predictive risk model for first-line antiretroviral therapy failure in South Africa. Journal of the International AIDS Society. J Int AIDS Soc. 2016;19:20897.

14. Vella C, Eymard-duvernay S, Sica J, Makosso L, Mongo AD, Boue V et al. Short report Virological failure rates and HIV-1 drug resistance patterns in patients on first-line antiretroviral treatment in semirural and rural Gabon. J Int AIDS Soc. 2012;VI:17985.

15. Antinori A, Coenen T, Costagiola D, Dedes N, Ellefson M, Gatell J et al. Late presentation of HIV infection: a consensus definition. HIV Med. 2011;12:61-4.

16. Huang A, Hogan JW, Luo X, DeLong A, Saravanan S WY et al. Global Comparison of Drug Resistance Mutations After First-Line Antiretroviral Therapy Across Human Immunodeficiency Virus-1 Subtypes. Open Forum Infect Dis.

17. Dadi TL, Kefale AT, Mega TA et al. Efficacy and tolerability of tenofovir disoproxil fumarate based regimen as compared to zidovudine based regimens: a systematic review and meta-analysis. AIDS Res Treat. 2017;2017:1-7.

18. Gunda DW. Prevalence and Predictors of Virological Failure among Adults HIV Patients Receiving First Line ART in Northwestern Tanzania: A Cross Sectional Study. EC Microbiol. 2019;15:683-90.

19. Ndakala FN, Oyugi JO, Oluka MA, Kimani J BG. The incidence of first-line antiretroviral treatment changes and related factors among HIV-infected sex workers in Nairobi, Kenya. Pan Afr Med J. 2017;28.

20. Shroufi A, Van Cutsem G, Cambiano V, Bansi-Matahru L DK et al. Simplifying switch to second-line antiretroviral therapy in sub Saharan Africa: predicted effect of using a single viral load to define efavirenz-based first-line failure. AIDS. 2019;33:1635-1644.

21. Koigi P, Ngayo MO, Khamadi S, Ngugi C NA. HIV type 1 drug resistance patterns among patients failing first and second line antiretroviral therapy in Nairobi, Kenya. BMC Res Notes. 2014;7:980.

22. Gupta RK, Gregson J, Parkin N, Haile-Selassie H, Tanuri A, Forero LA, et al. HIV-1 drug resistance before initiation or re-initiation of first-line antiretroviral therapy in low-income and middle-income countries: a systematic review and meta-regression analysis. Lancet Infect Dis. 2018;18:346-55.

23. Beck IA, Levine M, McGrath CJ, Bii S, Milne RS, Kingoo JM SI et al. Pre-treatment HIV-drug resistance associated with virologic outcome of first-line NNRTI-antiretroviral therapy: A cohort study in Kenya. EclinicalMedicine. 2020;18.

24. Petersen ML, van der Laan MJ, Napravnik S, Eron JJ, Moore RD, Deeks SG et al. Long-term consequences of the delay between virologic failure of highly active antiretroviral therapy and regimen modification. AIDS. 2008;22:2097-106.

25. Inzaule SC, Kityo CM, Siwale M, Akanmu AS WM et al. Previous antiretroviral drug use compromises standard first-line HIV therapy and is mediated through drug-resistance. Nat Sci Reports. 2018;8:15751. 
26. Nega J, Taye S, Million Y, Rodrigo C ES. Antiretroviral treatment failure and associated factors among HIV patients on frst-line antiretroviral treatment in Sekota, northeast Ethiopia. AIDS Res Ther [Internet]. 2020;17:39. Available from: https://doi.org/10.1186/s12981-020-00294-z

27. Agegnehu CD, merid MW YM. Incidence and predictors of virological failure among adult HIV patients on firstline antiretroviral therapy in Amhara regional referral hospitals; Ethiopia: a retrospective follow-up study. BMC Infect Dis [Internet]. 2020;20. Available from: https://doi.org/10.1186/s12879-020-05177-2

28. Ahmed M, Merga H JH. Predictors of virological treatment failure among adult HIV patients on firstline antiretroviral therapy in Woldia and Dessie hospitals, Northeast Ethiopia: a case-control study. BMC Infect Dis [Internet]. 2019;19. Available from: https://doi.org/10.1186/s12879-019-3924-4

29. Hassan AS, Nabwera HM, Mwaringa SM, Obonyo CA, Sanders EJ et al. HIV-1 virologic failure and acquired drug resistance among first-line antiretroviral experienced adults at a rural HIV clinic in coastal Kenya: a cross-sectional study. AIDS Res Ther [Internet]. 2014;11. Available from: http://www.aidsrestherapy.com/content/11/1/9

30. Peltzer K and Pengpid S. Socioeconomic Factors in Adherence to HIV Therapy in Low- and Middleincome Countries. J Heal Popul NUTR. 2013;13:150-70.

31. Ataro Z, Motbaynor B, Weldebreal F, Sisay M, Tesfa T, Mitiku H MD et al. Magnitude and causes of first-line antiretroviral therapy regimen changes among HIV patients in Ethiopia: a systematic review and meta-analysis. BMC Pharmacol Toxicol [Internet]. 2019;20. Available from: https://doi.org/10.1186/s40360-019-0361-3

32. Phillips AE et al. A systematic review and meta-analysis of quantitative interviewing tools to investigate self-reported HIV and STI associated behaviours in low- and middle-income countries. Int J Epidemiol. 2010;39:1541-55.

33. Mulisa D, Tolossa T, Wakuma B, Etafa W YG. Magnitude of first line antiretroviral therapy treatment failure and associated factors among adult patients on ART in South West Shoa, Central Ethiopia. PLoS One [Internet]. 2020;15:e0241768. Available from: https://doi.org/10.1371/journal. pone.0241768

34. Bezabih YM, Beyene F BW. actors associated with first-line antiretroviral treatment failure in adult HIV positive patients: a case-control study from Ethiopia. BMC Infect Dis [Internet]. 2019;19. Available from: https://doi.org/10.1186/s12879-019-4170-5

35. Bezabih YM, Beyene F, Bezabhe WM. Factors associated with first-line antiretroviral treatment failure in adult HIV- positive patients: a case-control study from Ethiopia. BMC Infectious Diseases; 2019;18.

36. Kwobah CM, mwangi AW, koech JK, Simiyu GN SA. Factors Associated with First-Line Antiretroviral Therapy Failure amongst HIV-Infected African Patients: A Case-Control Study. World J AIDS. 2012;2:271-8.

37. Grangeiro A, Escuder MM, Menezes PR, Alencar R A de CE. Late Entry into HIV Care: Estimated Impact on AIDS Mortality Rates in Brazil, 2003-2006. PLoS One. 2011;6:e14585. 
38. Drain PK, Losina E, Parker G, Giddy J, Ross D et al. Risk Factors for Late-Stage HIV Disease Presentation at Initial HIV Diagnosis in Durban, South Africa. PLoS One. 2013;8:e55305.

39. Chen Q, Zeng D, She Y, Lyu Y GX et al. Different transmission routes and the risk of advanced HIV disease: A systematic review and network meta-analysis of observational studies. EclinicalMedicine [Internet]. 2019; Available from: https://doi.org/10.1016/j.eclinm.2019.10.003

40. Dai S-Y, Liu J-J, Fan Y-G, Shan G-S ZH-B et al. Prevalence and Factors Associated With Late HIV Diagnosis. J Med Virol. 2015;87:970-977.

41. Deblonde J, De Koker P, Hamers FF, Fontaine J, Luchters S TM. Barriers to HIV testing in Europe: a systematic review. Eur J Public Health. 20:422-32.

42. Aldrete S, Jang JH, Easley KA, Okulicz J, Dai T, Chen YN et al. CD4 rate of increase is preferred to CD4 threshold for predicting outcomes among virologically suppressed HIVinfected adults on antiretroviral therapy. PLoS One [Internet]. 2020;15:e0227124. Available from: https://doi.org/10.1371/journal. pone.0227124

43. Chilot Desta Agegnehu MWM and MKY. Incidence and predictors of virological failure among adult HIV patients on firstline antiretroviral therapy in Amhara regional referral hospitals; Ethiopia: a retrospective follow-up study. BMC Infect Dis. 2020;20.

\section{Figures}




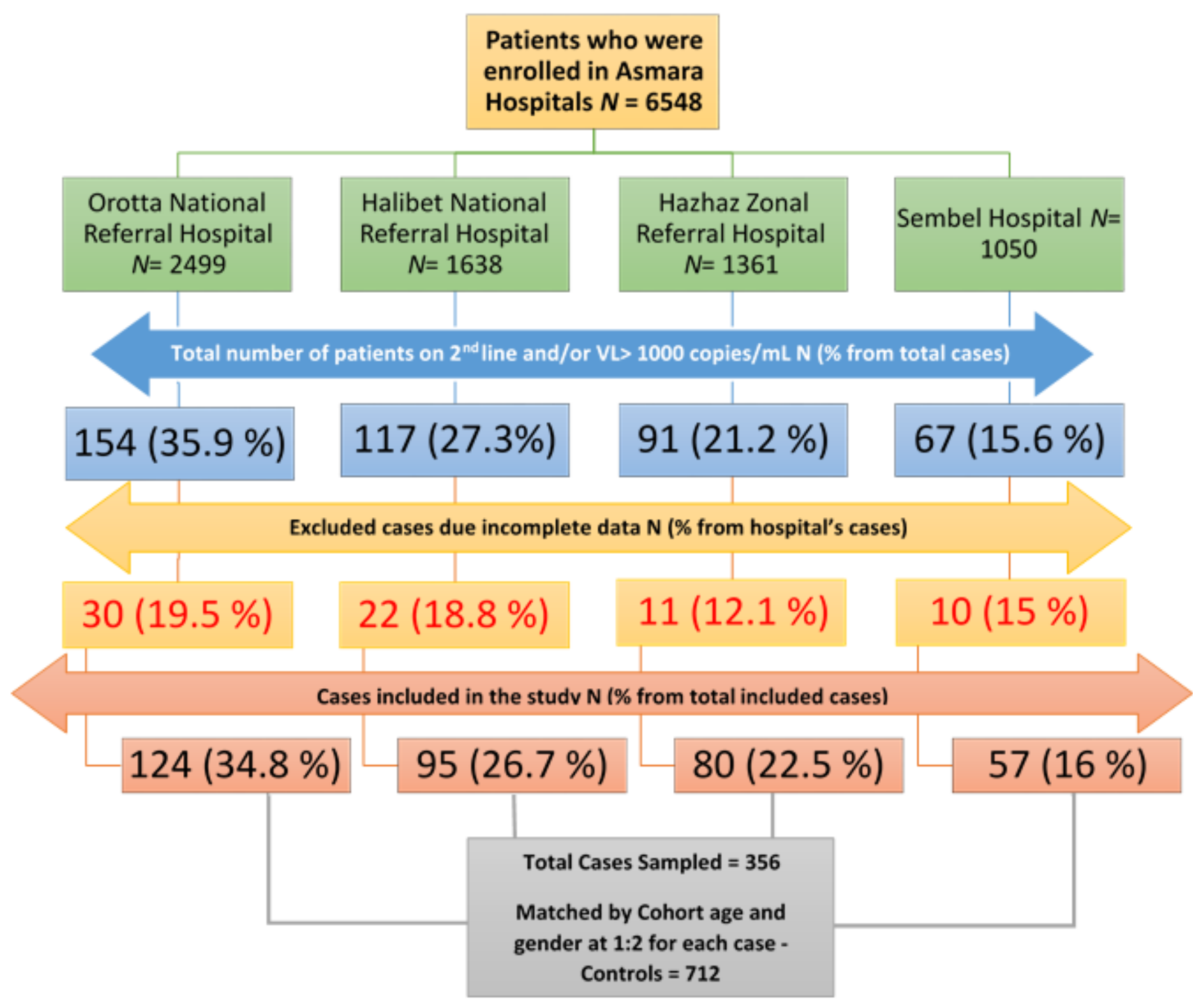

Figure 1

Flow diagram of study participants' recruitment Outcomes 


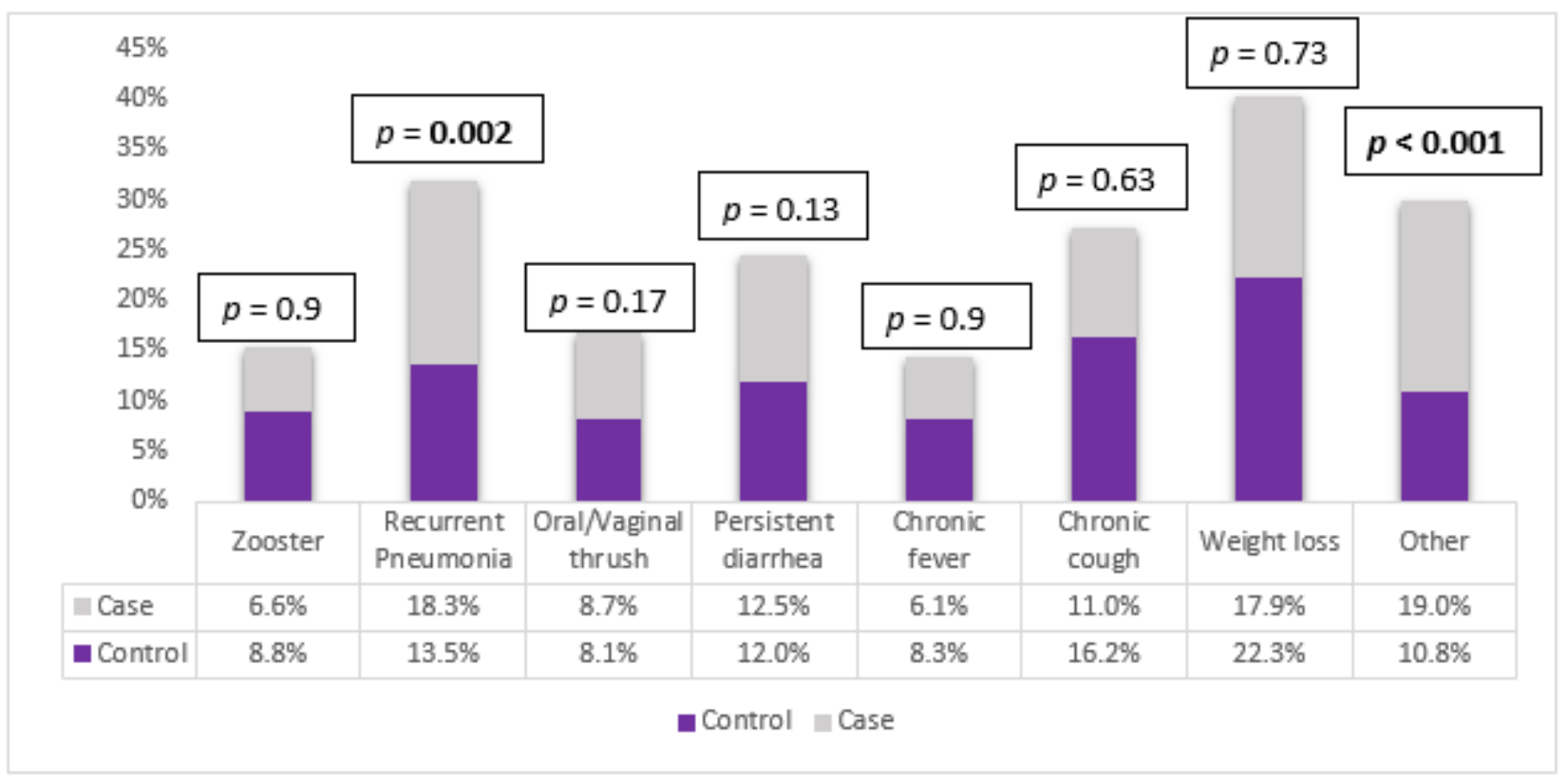

Figure 2

Frequency of specific comorbidities among patients

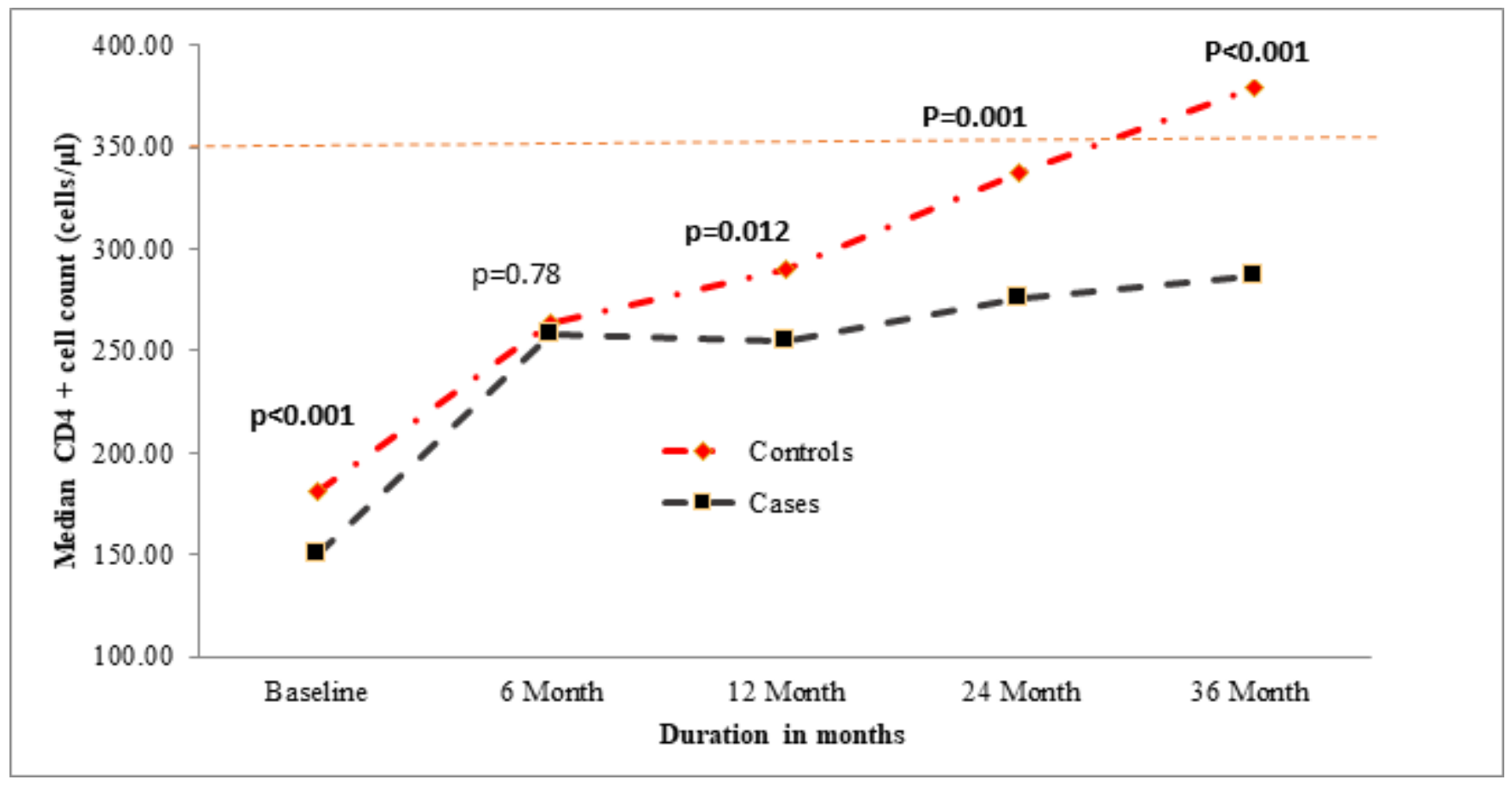

Figure 3

Graph showing the median $\mathrm{CD} 4^{+}$cell count at baseline and different periods of treatment, stratified by Therapy outcome status. 